\title{
Proceso profesionalizante: Una intervención en enseñanza superior
}

\author{
A Professionalizing Process: An Intervention in Higher Education \\ Processo de profissionalização: uma intervenção no ensino superior
}

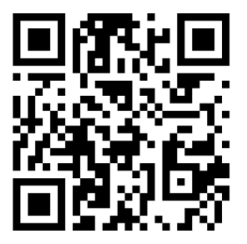

\author{
Minerva Camacho-Javier \\ Universidad Juárez Autónoma de Tabasco \\ División de Ciencias Económico Administrativas \\ Tabasco, México \\ minerva.camacho@ujat.mx \\ https://orcid.org/0000-0003-2655-2092 \\ José César López-del Castillo \\ Universidad Juárez Autónoma de Tabasco \\ División de Ciencias Económico Administrativas \\ Tabasco, México \\ cesar.lopez@ujat.mx \\ https://orcid.org/0000-0001-9454-9093
}

Recibido • Received • Recebido: 01 / 08 / 2020

Corregido • Revised • Revisado: 30 / 06 / 2021

Aceptado • Accepted • Aprovado: 09 / 10 / 2021

\begin{abstract}
Resumen:
Introducción. En México es palpable la falta de convergencia educativa en la enseñanza superior, con un avance desigual en la formación por competencias, sobre todo al sur del país. Se considera que el personal docente es el eje principal en el desarrollo de la calidad educativa. El propósito de esta investigación es conocer cómo se asume un proceso profesionalizante que busca el desarrollo de las competencias docentes aplicado a un grupo de maestros y maestras de una institución pública de nivel superior ubicada al sur del país. Metodología. Se realizó una investigación-acción colaborativa y participativa longitudinal de tipo exploratoria-descriptiva. Se diseñó un proceso profesionalizante y se aplicó en una de las divisiones académicas más pobladas de la institución educativa (IE) sujeto/objeto de estudio. Esta fue desarrollada en un período de año y medio con un grupo de docentes seleccionado por conveniencia y con roles intercambiables. Resultados. A través de indicadores cualitativos y cuantitativos, se muestra el involucramiento del personal docente en el proceso profesionalizante. Estos indicadores son: participación voluntaria; reconocimientos; difusión de la actuación académica; evidencias de la enseñanza aprendizaje por competencias; interacción didáctico-pedagógica y la teoría en la práctica. Discusión. Se destaca la importancia de una gestión educativa con liderazgo proactivo que practique un currículo más cohesionado hacia un trabajo interdisciplinar y transdisciplinar, en procura de una gestión socialmente responsable. Finalmente, la investigación acción colaborativa y participativa guía hacia un saber-puente para el desarrollo de las competencias docentes; sin embargo, se requiere la continuidad de este proceso para innovar en la educación superior.
\end{abstract}

Palabras claves: Competencias docentes; investigación acción colaborativa y participativa; proceso profesionalizante. 
http://doi.org/10.15359/ree.26-1.6

http://www.una.ac.cr/educare

educare@una.ac.cr

\begin{abstract}
:
Introduction. The lack of educational convergence in higher education is palpable in Mexico, with uneven progress in training by competencies, especially in the country's south. The teaching staff is considered to be the main axis in the development of educational quality. Therefore, this research aims to know how a professionalizing process applied to a group of male and female teachers from a public higher education institution located in the south of the country is assumed, seeking the development of teaching competencies. Methodology. A collaborative and participatory longitudinal exploratory-descriptive action research was conducted. A professionalizing process was designed and applied in one of the most populated academic divisions of the educational institution (IE) that was the subject and object of the study. The research was conducted in one and a half years with a group of teachers selected for convenience and with interchangeable roles. Results. Through qualitative and quantitative indicators, the involvement of the teaching staff in the professionalizing process is shown. These indicators are voluntary participation, recognitions, dissemination of academic performance, evidence of teaching-learning by competencies, didactic-pedagogical interaction, and theory in practice. Discussion. The importance of an educational management with proactive leadership that practices a more cohesive curriculum towards interdisciplinary and transdisciplinary work is highlighted, seeking socially responsible management. Finally, collaborative and participatory action research guides towards a knowledge bridge for the development of teaching competencies; however, the continuity of this process is required to innovate in higher education.
\end{abstract}

Keywords: Teaching skills; collaborative and participatory action research; professionalizing process.

\title{
Resumo:
}

Introdução. No México, é palpável a falta de convergência educacional no ensino superior, com avanços desiguais na formação por competências, especialmente no sul do país. O corpo docente é considerado o principal eixo no desenvolvimento da qualidade educacional. O objetivo desta pesquisa é conhecer como se assume um processo profissionalizante aplicado a um grupo de docentes de uma instituição pública de nível superior localizada no sul do país, visando o desenvolvimento de competências docentes. Metodologia. Foi realizada uma pesquisa-ação exploratória-descritiva longitudinal colaborativa e participativa. Um processo de profissionalização foi desenhado e aplicado em uma das divisões acadêmicas mais populosas da Instituição Educativa (IE) sujeito/objeto de estudo. Este trabalho foi desenvolvido em um período de um ano e meio com um grupo de docentes selecionados por conveniência e com funções variadas. Resultados. Por meio de indicadores qualitativos e quantitativos, evidencia-se o envolvimento do corpo docente no processo de profissionalização. Esses indicadores são: participação voluntária; reconhecimentos; divulgação do desempenho acadêmico; evidências de ensino-aprendizagem por competências; interação didático-pedagógica e teoria na prática. Discussão. Destaca-se a importância de uma gestão educacional com lideranças proativas que pratiquem um currículo mais coeso no sentido de um trabalho interdisciplinar e transdisciplinar, buscando uma gestão socialmente responsável. Por fim, a pesquisa-ação colaborativa e participativa orienta para uma ponte de conhecimento para o desenvolvimento de competências docentes; no entanto, a continuidade desse processo é necessária para inovar no ensino superior.

Palavras-chave: Habilidades de ensino; pesquisa-ação colaborativa e participativa; processo de profissionalização. 


\section{Introducción}

De acuerdo con la Asociación Nacional de Universidades e Instituciones de Educación Superior (Asociación Nacional de Universidades e Instituciones de Educación Superior [ANUIES], 2018), México debe trabajar en una mejor instrumentación pedagógica, si quiere avanzar y ser parte de la sociedad de la información y el conocimiento.

El énfasis continúa en aquellos Estados con mayor rezago social y económico. Al respecto, el sur de la República Mexicana es donde se tiene mayor atraso educativo, además de una preparación profesional que limita el desarrollo del país (Arechavala Vargas y Sánchez Cervantes, 2017).

La falta de convergencia educativa en la enseñanza superior y el avance desigual en la formación por competencias indica: (1) escaso trabajo colegiado que transite hacia una cultura colaborativa (Aguilar Morales et al., 2013); (2) gestión que facilite y promueva el intercambio de experiencias y el trabajo autorreflexivo en el desempeño didáctico-pedagógico (Magaña Medina y Aquino Zúñiga, 2014) y (3) el involucramiento y reconocimiento a la labor docente (Magaña Medina y Sánchez Esobedo, 2013).

Entonces, ¿desde qué mirada se debe observar la enseñanza profesional? Algunos estudios, como el de Villarroel y Bruna (2017), destacan que la persona docente de excelencia tiene que ver más con el estilo que muestra en el dominio del conocimiento, la forma de comunicarlo y las actitudes manifestadas, en comparación con las competencias específicas que posea; sin embargo, también se señala que la calidad del aprendizaje por competencias recae en la labor que se lleva a cabo en la enseñanza cuando esta muestra una construcción innovadora, coherente y organizada del conocimiento a mostrar.

Derivado de lo anterior, se genera un discurso sobre la importancia de la profesionalización docente como parte de la mejora en la calidad educativa (Aberbuj et al., 2019; Garduño, 2019; Ramírez-Díaz, 2020; Vaillant, 2016), sin olvidar que los hábitos y creencias adquiridos en nivel superior no son fáciles de erradicar. Díaz-Barriga y Rigo (2009) reflexionan sobre el papel del personal docente y el impacto ante nuevas formas de enseñanza que pretendan modificar el acto educativo ya que, lo nuevo, es tomado como arbitrariedad ante la costumbre de la propia enseñanza. Por otro lado, cuando existe apoyo y reconocimiento por parte de la institución educativa, el personal docente muestra disposición para arriesgarse a experimentar.

El propósito de este artículo es conocer cómo se responde ante una intervención mediante un proceso profesionalizante en una institución pública de nivel superior ubicada al sur del país.

\section{Elementos de estudio}

\section{El entorno}

Tabasco es una entidad federativa ubicada al sur de México, donde se encuentra la Universidad Juárez Autónoma de Tabasco (UJAT), entidad precursora de la educación superior 
http://doi.org/10.15359/ree.26-1.6

http://www.una.ac.cr/educare

educare@una.ac.cr

desde finales del siglo XIX. En la década de los 90, el Estado aumentó la oferta educativa, no así la calidad. A la fecha, la UJAT es una de las mejores opciones en educación superior.

Sin embargo, investigaciones realizadas por Lamoyi Bocanegra y Pintos Blancas (2018) resaltan que la gestión educativa ejercida en la UJAT requiere, entre otras cosas, relacionarse más con su planta docente para mejorar la calidad de su enseñanza.

\section{Divisiones académicas}

De 1985 a 1988, se estableció el primer Plan Institucional de Desarrollo, donde surge el modelo de organización matricial. De este plan se derivan las divisiones académicas. Estas se encuentran agrupadas en programas educativos por áreas del conocimiento. Hoy en día, la universidad cuenta con doce divisiones académicas y un Plan de desarrollo a largo plazo 2028 que, de manera general, pretende estar a la vanguardia de los constantes cambios manifestados en el entorno. Es así como la innovación educativa en la UJAT pretende ser mediada desde la pertinencia y factibilidad universitaria.

\section{Consideraciones de rol}

En avances de investigación presentados en el 2018, se describió el panorama general de una docencia con una diversidad de criterios y acciones académicas sobre el enfoque por competencias (Camacho Javier et al., 2018). Se identificaron dos desafíos educativos: (1) la gestión pedagógica para el desarrollo de una profesionalización docente y (2) el liderazgo en la gestión educativa, para direccionar al personal docente hacia un saber ser y un saber actuar más colaborativo y congruente dentro de un entorno que estila realidades diferentes.

Aunado a lo anterior, se reconoce que el actuar pedagógico parte de una relación proactiva entre los actores educativos de diferentes disciplinas; pero, para que se dé este tipo de dinámica se requiere de iniciativa directiva que norme o legitime espacios de aprendizaje profesional, con el objetivo de moldear buenas prácticas educativas para la innovación permanente en la didácticapedagógica (Castillo-Cedeño et al., 2016; Durán Rodríguez y Estay-Niculcar, 2016).

\section{Oportunidad y propósito de intervención}

El acto pedagógico, en sí, encierra una visión integral y continua entre la teoría y la práctica. Desde esta concepción se configuran los procesos didácticos y se reflexiona sobre el acto educativo.

Daniels (2003) esgrime que"si no comprendemos cómo se implementan en las instituciones las posibilidades para aprender, nuestros intentos de mejorar realmente la calidad se verán frustrados" (p. 15). Se infiere que el involucramiento pedagógico debe ser horizontal, asumido de manera consciente, razonada y sentida por todos los sujetos, y debe evitar el reduccionismo del acto educativo. 
http://doi.org/10.15359/ree.26-1.6

\section{Pregunta y alcance de investigación}

Al reflexionar sobre el rol que juega el personal docente-tutor y la importancia de la transversalidad institucional para incrementar experiencias colaborativas (Tejada Fernández, 2020), surge la pregunta de investigación: ¿Cómo asumen docentes de enseñanza superior el desarrollo de sus competencias docentes al trabajar de manera colaborativa y participativa? En consecuencia, surge el proyecto denominado Desarrollo de las competencias docentes en la investigación acción colaborativa.

El propósito de investigación es conocer cómo responde el personal docente ante un proceso profesionalizante ex profeso, donde el proyecto se considera desde un modelo de actuación interdisciplinar con la metodología de investigación acción colaborativa y participativa (Colmenares, 2012).

\section{Aproximación teórica}

Avanzar en el entendimiento de las competencias refiere, precisamente, a esa capacidad de instrumentación formativa que se da a través de un trabajo más integrado y participativo, donde el personal docente conozca, vivencie y desarrolle una forma de ser y de actuar más motivada y eficiente en la aplicación de una enseñanza-aprendizaje por competencias (Casanova Romero et al., 2018; Ramírez-Díaz, 2020).

Durante la investigación documental, se identificó que la ejecución participativa muestra un paralelismo metodológico con la propuesta socioformativa de Tobón (2017), cuyo enfoque se sustenta en algunos de los principios de la teoría del pensamiento complejo de Morin (1990), ya que se orienta hacia la mejora de los procesos educativos en la enseñanza y el aprendizaje integral y significativo por competencias.

La intención del proyecto, en similitud al trabajo de Tobón (2017), contempla mejorar los procesos educativos a través de una metodología de aprendizaje interactiva que lleva al desarrollo de competencias transversales y específicas.

Sin embargo, para saber aplicar la metodología del AOP, el personal docente de nivel superior debe poseer competencias docentes idóneas para este tipo de enseñanza y aprendizaje por competencias. Perrenoud (2004) propone las siguientes competencias docentes (CD) (ver Figura 1):

Figura 1: Las diez Competencias Docentes (CD) planteadas por Perrenoud

\begin{tabular}{|c|c|c|c|}
\hline $\begin{array}{l}\text { Saber planificar: } \\
\text { el qué y los cómo de la enseñanza. }\end{array}$ & $\begin{array}{c}\text { Saber actuar: } \\
\text { actividades de aprendizaje. }\end{array}$ & $\begin{array}{c}\text { Saber interactuar: } \\
\text { contextualizar el aprendizaje. }\end{array}$ & $\begin{array}{l}\text { Saber ser docente: } \\
\text { garantizar el éxito y bienestar profesional }\end{array}$ \\
\hline $\begin{array}{l}\text { CD 1: Establecer objetivos y situaciones } \\
\text { de aprendizaje. } \\
\text { CD 2: Planificar la progresión del } \\
\text { aprendizaje/evaluar. }\end{array}$ & $\begin{array}{l}\text { CD 3: Gestionar el aula. } \\
\text { CD 4: Atender a la diversidad. } \\
\text { CD 5: Educar en valores. } \\
\text { CD 6: Usar las TIC. }\end{array}$ & $\begin{array}{l}\text { CD 7: Trabajar en equipo y participar en } \\
\text { la gestión institucional. } \\
\text { CD 8: Conocer la comunidad y entorno } \\
\text { escolar, implicarlos e informarlos. }\end{array}$ & $\begin{array}{l}\text { CD 9: Gestionar el propio trabajo y } \\
\text { tomar decisiones de mejora. } \\
\text { CD 10: Competencias curriculares. }\end{array}$ \\
\hline
\end{tabular}


http://doi.org/10.15359/ree.26-1.6

http://www.una.ac.cr/educare

educare@una.ac.cr

Tobón (2017) y Perrenoud (2004) coinciden en que el personal docente debe saber colaborar (interactuar), saber mediar el aprendizaje (planificar y actuar), saber evaluar el aprendizaje (planificar) y saber gestionar los recursos y el manejo de las TIC (actuar, interactuar y saber ser docente); sin embargo, Tobón (2017) trasciende su visión hacia dos aspectos: al saber comunicar y sobre el currículo. El primero no solo implica el saber comunicar de manera clara en un entorno educativo, sino que apela al saber comunicar científico de cada docente y, sobre el segundo, pasa de un paradigma constructivista centrado en una didáctica de grupos pequeños dentro de un espacio áulico a un enfoque socioformativo que implica el desarrollo de aprendizajes colaborativos en diferentes contextos: educativo, social y organizacional, aproximándose un poco a la concepción pedagógica de Freire (2006).

\section{Método}

Se realizó una investigación-acción colaborativa y participativa longitudinal (de agosto 2018 a enero de 2020) (Bisquerra Alzina, 2009) de tipo exploratoria-descriptiva.

\section{Sujetos participantes}

La estrategia metodológica determinó el papel de las personas investigadoras y el nivel de involucramiento con el personal docente participante.

En la selección de los sujetos participantes se aplicó el muestreo por conveniencia (Hernández Sampieri et al., 2014), se integraron a algunos sujetos docentes que tuvieran un doble rol, como se muestra en la Tabla 1.

En el proceso se utilizó el método secuencial (Creswell, 2008), comenzando con un grupo pequeño de sujetos $y$, posteriormente, ampliando la participación a un grupo más grande, con un total de participación de 13 sujetos (ver Tabla 1). Como dato secundario y para posteriores estudios, se indica que durante el período abarcado se tuvo un alcance aproximado de 870 estudiantes.

Tabla 1: Características de los sujetos participantes

\begin{tabular}{llccc}
\hline \multicolumn{1}{c}{ Tipo de rol } & Conocimiento disciplinar & Rango de edad & \multicolumn{2}{c}{ \# participantes por sexo } \\
\cline { 3 - 5 } & & & F & M \\
\hline Personal docente y administrativo & Administración y finanzas & De 33 a 68 & 4 & 2 \\
Personal docente & $\begin{array}{l}\text { Economía, mercadotecnia y } \\
\text { contabilidad }\end{array}$ & De 35 a 70 & 1 & 3 \\
$\begin{array}{l}\text { Personal docente colaborador e } \\
\text { investigador }\end{array}$ & $\begin{array}{l}\text { Mercadotecnia, estudios } \\
\text { organizacionales, psicoterapia } \\
\text { guestalt y educación }\end{array}$ & De 50 a 58 & 2 & 1 \\
\hline \multicolumn{1}{c}{ Total } & & & \\
\hline
\end{tabular}

Nota: Elaboración propia. Datos obtenidos en las entrevistas individuales para participar en el proyecto.

6 
http://doi.org/10.15359/ree.26-1.6

http://www.una.ac.cr/educare

\section{Instrumentos y técnicas}

Las preguntas generadas, a partir de la pregunta principal, guían el proceso de investigación (ver Figura 2), donde prevalece el abordaje cualitativo.

Figura 2: Técnicas, recursos y preguntas de investigación

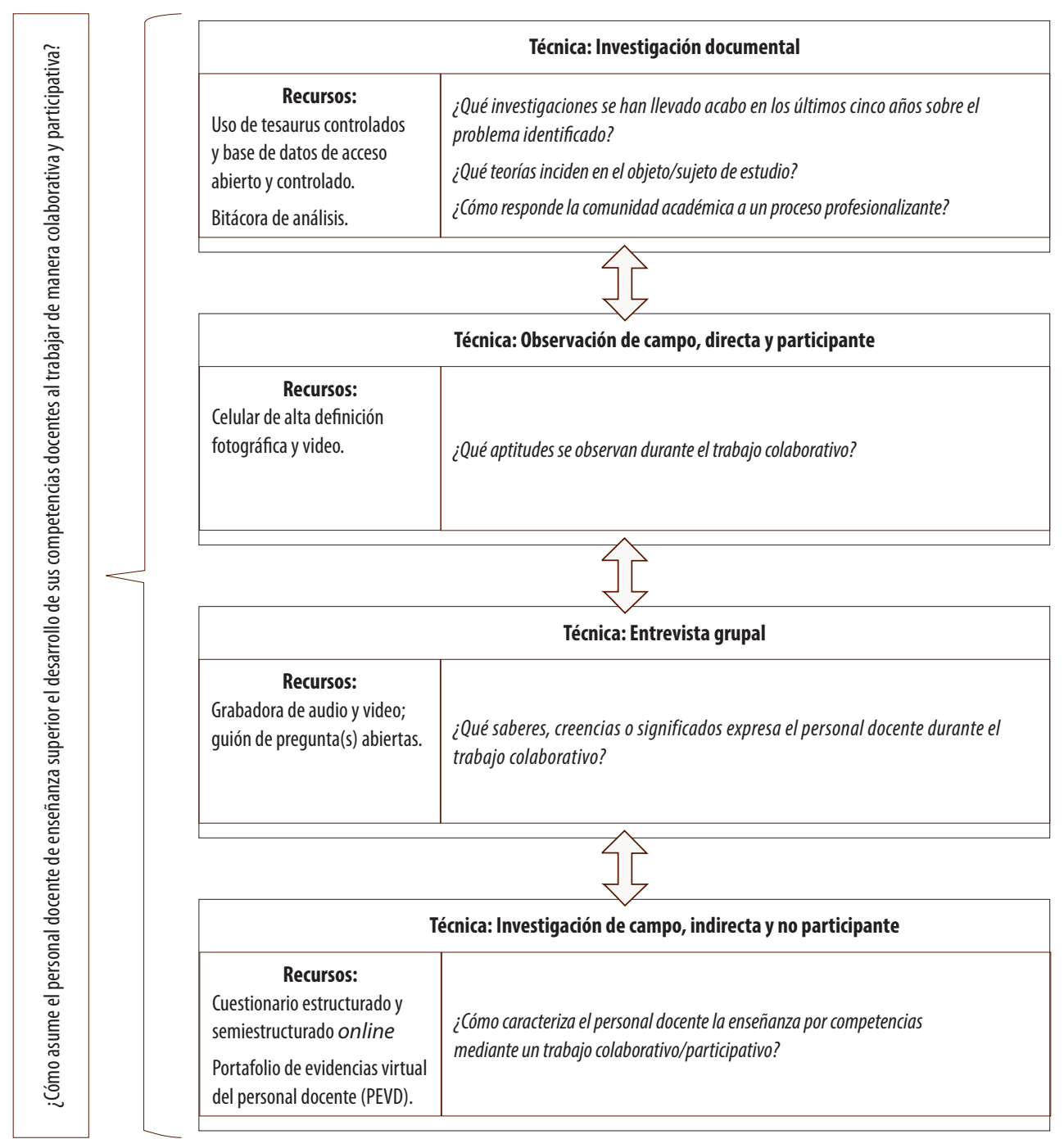

Nota: La figura muestra las técnicas y recursos utilizados en la investigación documental y de campo. Estos fueron aplicados durante los momentos de interacción grupal con el personal docente participante, tanto en sesiones presenciales (tipo focus group) como en línea (encuestas diseñadas con la plataforma Google Drive). Elaboración propia.

\section{Del procesamiento el análisis de datos teóricos}

Para la organización, sistematización y análisis de la información teórica, se usó el ATLAS.ti v.9. A partir de diversas entidades y la codificación abierta, emergieron categorías 
http://doi.org/10.15359/ree.26-1.6

http://www.una.ac.cr/educare

educare@una.ac.cr

teóricas; de la misma manera, se identificaron las co-currencias de las entrevistas durante las sesiones grupales.

\section{Procedimiento}

\section{Del proceso profesionalizante}

El inicio del proyecto es marcado por una postura reflexiva sobre la importancia de atender lo dictado por organismos internacionales, la valoración realizada por la ANUIES, así como los cambios presentados en el modelo educativo de la institución educativa (IE), objeto/sujeto de estudio.

De esta manera, la preparación hacia el desarrollo de las competencias docentes es reconocida por el grupo de investigación como un caminar en un proceso administrativo, al buscar generar una gestión educativa cíclica que lleve a la a pertura de encuentros experienciales pensados desde la contextualización y realidad vivida de los sujetos de estudio, para guiar hacia posibilidades de cambio pedagógico más eficientes y saludables (Castillo-Cedeño et al., 2016). A finales del 2017 se comienza a bosquejar un proceso de profesionalización integral y autorregulador (Ver Figura 3).

Figura 3: Proceso profesionalizante

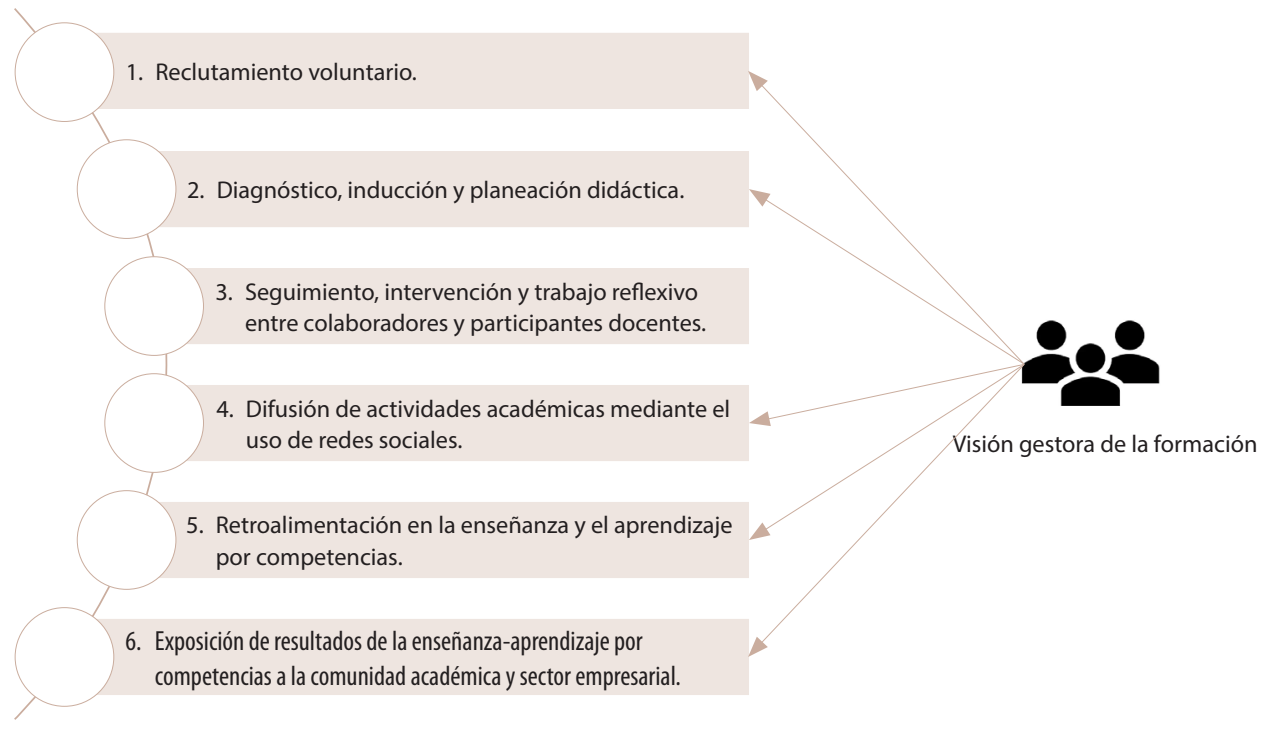

Nota: Muestra el proceso de intervención colaborativa y participativa aplicado en cada uno de los ciclos de los años trabajados y denominado proceso profesionalizante. Se aclara que la visión gestora refiere a la importancia del acompañamiento por el área administrativa responsable en el seguimiento y apoyo académico.

Elaboración propia.

Es importante distinguir que la denominación "proceso profesionalizante" determinada para este estudio tiene que ver con la innovación de procesos de calidad buscados en la formación educativa por competencias (Cañón Rodriguez, 2003). 
http://doi.org/10.15359/ree.26-1.6

El registro del proyecto inició a mediados del 2018, momento en donde solo estaba disponible la opción sin financiamiento. Otro punto por resaltar son los cambios en las áreas administrativas. Derivado de lo anterior, se detuvo la revisión y dictaminación positiva hasta finales del mismo año para finalizar en enero de 2021 (el proyecto solo se pudo avanzar hasta enero de 2020 por causa de la pandemia causada por el COVID-19); se mantuvo la opción sin financiamiento, lo cual obligó a hacer ajustes en los objetivos y a sujetar el alcance a proyectos académicos con participación local y asistencia reducida a congresos nacionales o internacionales.

El estudio comenzó una interacción interdisciplinar en una de las divisiones académicas más pobladas de la institución educativa (IE). El desarrollo de las etapas diagnóstico, inducción y planeación didáctica, y seguimiento e intervención se muestran en la Figura 4. Se llevó un registro detallado con un diario de trabajo tipo minuta durante cada uno de los ciclos y durante los años abarcados (de agosto 2018 a enero de 2020), se realizó un total de tres ciclos largos (1 ciclo = 6 meses); no se pudo preparar el cuarto evento en enero de 2020 a causa de la pandemia, como fue mencionado en el párrafo anterior.

Figura 4: Preparación, aplicación, gestión y seguimiento de las competencias docentes

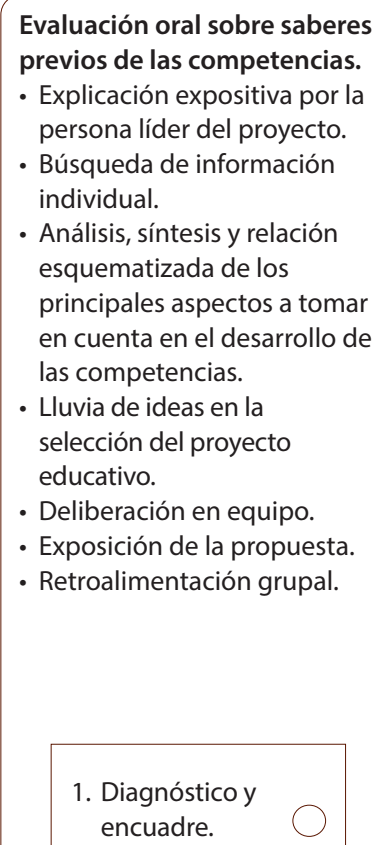
previos de las competencias.

- Explicación expositiva por la persona líder del proyecto.

- Búsqueda de información individual.

- Análisis, síntesis y relación esquematizada de los principales aspectos a tomar en cuenta en el desarrollo de las competencias.

- Lluvia de ideas en la selección del proyecto educativo.

- Deliberación en equipo.

- Exposición de la propuesta.

- Retroalimentación grupal.

1. Diagnóstico y encuadre.

Exposición de la persona líder del proyecto sobre la planeación didáctica.

- Distribución de acciones (roles-actividades) para diseño de la planeación didáctica.

- Diseño del entorno virtual del docente.

- Búsqueda, revisión, recopilación y archivado digital de material electrónico-documental para las asignaturas en el desarrollo del aprendizaje por competencias.

- Gestión administrativa: solicitudes de espacio, permisos, visitas, entrevistas y exposición final de la enseñanza-aprendizaje por competencias.

- Diseño de herramientas digitales como guías tutoriales en la presentación o comunicación del proyecto.

- Diseño de formatos de evaluación (rúbricas y listado de cotejo) para las etapas de aprendizaje formativo y sumativo.

- Elaboración de la planeación didáctica bajo el aprendizaje orientado a proyectos (AOP).

- Retroalimentación grupal.

\section{Diseño de la} planeación didáctica.
- Reuniones asincrónicas por medio de la plataforma Google Drive.

- Reuniones presenciales en los espacios asignados por la coordinación de docencia.

- Utilización de redes sociales (facebook e instagram) para la socialización del avance académico por competencias.

3. Gestión y seguimiento.

Nota: Elaboración propia. Datos obtenidos de la inducción al método de enseñanza mediante un curso-taller impartido al personal docente de la institución. 
http://doi.org/10.15359/ree.26-1.6

http://www.una.ac.cr/educare

educare@una.ac.cr

\section{De las personas investigadoras participantes}

La intervención se llevó a cabo con el Grupo de Investigación (Gl) Gestión e Innovación Socioeducativa, con la finalidad de generar pericia en la metodología aplicada y afianzar el avance hacia cuerpo académico (CA).

En este sentido, se buscaron estrategias de intervención socioeducativa para generar conocimientos teórico-prácticos en el marco del desarrollo de competencias docentes: (a) liderazgo en procesos educativos de aprendizaje interdisciplinar, que involucra el trabajo cooperativo y colaborativo, (b) capacidad para diseñar o innovar en entornos de aprendizaje mixtoy (c) el cultivo permanente del pensamiento crítico-reflexivo sobre el actuar socioeducativo. De este modo, los integrantes del Gl se involucraron con el personal docente participante.

\section{Del personal docente participante}

El reclutamiento de quienes participaron fue por la vía voluntaria y por invitación, al presuponer una necesidad de aprendizaje. Sin restricción de edad y sexo; con poco o ningún conocimiento sobre una enseñanza por competencias y manejo de herramientas virtuales.

El reclutamiento, tanto del primer grupo como del segundo, se realizó en la Sala de Maestros de la División. Se explicó el propósito y los beneficios académicos y de formación que podrían obtener a corto y mediano plazo con el trabajo interdisciplinario.

\section{Consideraciones del entorno}

El entorno seleccionado fue una división ubicada fuera de la ciudad, donde se encuentran los primeros y segundos ciclos de diversas licenciaturas.

Las instalaciones de la división cuentan con espacios amplios, bien iluminados y climatizados; mesas y sillas para trabajo en equipo; proyector y conexión a internet. El internet presenta fallas en la señal y solo el personal docente puede conectarse gratuitamente, mientas que el estudiantado debe pagar para acceder al servicio.

En este apartado se consideran las presentaciones del proyecto a los diferentes sujetos directivos (tres cambios de dirección durante su desarrollo), para gestionar su apoyo (permisos, oficios, espacio de reunión, tramitación de espacios para eventos, invitaciones a personas externas, entre otros).

\section{Resultados}

Partiendo de la pregunta de investigación y de la integración de las preguntas secundarias (ver Figura 2), se muestran los resultados obtenidos y delimitados por indicadores cualitativos y cuantitativos, que aluden al involucramiento del personal docente en el proceso profesionalizante. Estos son: participación voluntaria, reconocimientos, difusión de la actuación 
http://doi.org/10.15359/ree.26-1.6 http://www.una.ac.cr/educare educare@una.ac.cr

académica, evidencias de la enseñanza aprendizaje por competencias, interacción didácticopedagógica y la teoría en la práctica.

A continuación, se detalla cada uno de estos:

\section{Participación voluntaria}

El personal docente con ambos roles (docente-administrativo), sin considerar a las personas colaboradoras, tuvo cambios de rol durante el proceso. Lo anterior se debió a los cambios en la dirección de la división (tres durante la realización del proyecto); ello afectó la continuidad de este en las gestiones.

La situación anterior impactó en la percepción del personal docente participante, aunado a la propia resistencia al cambio (Díaz Barriga y Rigo, 2009) con respecto a todo lo que implica planear y preparar una enseñanza-aprendizaje por proyecto.

En relación con el desarrollo de la investigación, la Figura 5 muestra el lenguaje involucrado en el discurso de los sujetos docentes participantes con respecto al propio sentir durante el proceso que se estaba llevando de forma guiada, donde la palabra "entorno"fue la más señalada.

Figura 5: Co-currencias del discurso vivo

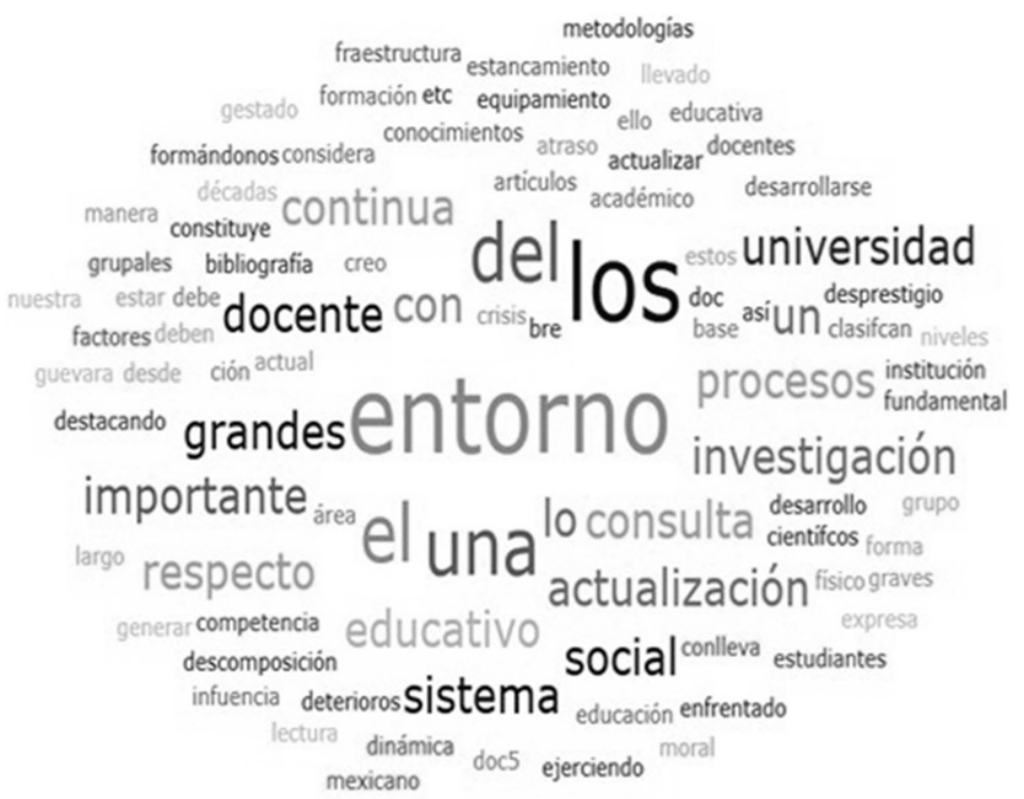

Nota: Palabras con mayores repeticiones durante las sesiones grupales de edificación y reflexión pedagógica.

Elaboración propia a partir del software ATLAS.ti v.9. 
http://doi.org/10.15359/ree.26-1.6

http://www.una.ac.cr/educare

educare@una.ac.cr

\section{Reconocimientos}

Los reconocimientos para el personal docente participante se otorgaron por: (1) cursos-talleres con duración de $40 \mathrm{hrs}$. sobre el desarrollo de competencias docentes en el diseño de planeaciones didácticas; (2) constancia como ponentes en la 1. ${ }^{\text {ra Exposición }}$ de Resultados Académicos por Competencias; (3) constancia por haber coordinado la 1. ${ }^{\text {, }}$, $2{ }^{\text {da }}$ y 3 ra $^{\text {ra }}$ exposición de resultados académicos; (4) por el diseño de entornos virtuales con la Web 2.0; (5) por la elaboración de material digital para una enseñanza b-learning; (6) por el diseño e impartición de diplomado por competencias en entornos mixtos; (7) por participación en el diplomado semipresencial en Formación en Competencias Docentes para un Entorno Mixto de 120 hrs. (ver Tabla 2).

Tabla 2: Número de reconocimientos otorgados por actividad

\begin{tabular}{lcc}
\hline \multirow{2}{*}{ Rubro profesionalizante } & \multicolumn{2}{c}{ Número de constancias } \\
\cline { 2 - 3 } & 2018 & 2019 \\
\hline Elaboración curso-taller & 1 & Ciclo-01 y 02 \\
Participación en curso-taller & 5 & - \\
Diseño e impartición de diplomado & - & 6 \\
Participación en diplomado & - & 11 \\
Ponente en exposición de resultados & 5 & 12 \\
Organizador de evento & 5 & - \\
Diseño de entorno virtual & 5 & - \\
\hline
\end{tabular}

Nota: Los datos corresponden a las actividades realizadas durante agosto de 2018 a enero de 2020. El evento programado para el 1er ciclo de 2020 se canceló debido a la pandemia por COVID-19. Por lo que los datos registrados abarcan solo tres ciclos largos. Elaboración propia. 
http://doi.org/10.15359/ree.26-1.6

Se puede observar en la Tabla 2 que, entre el 2018 y el 2019, destacan dos aspectos significativos: (1) aumento de las personas docentes participantes y (2) el interés por involucrarse en las actividades del proyecto. Esto se relaciona con lo expuesto en el primer indicador participación voluntaria y compromiso.

\section{Difusión de la actuación académica}

Este proceso contempló dos momentos de socialización:

1. Proceso de enseñanza-aprendizaje áulico. Presentación del equipo de docentes participantes y la metodología del AOP con cada grupo. Se proyectó el problema y la planeación del trabajo interdisciplinario; se señaló la participación de personas invitadas externas para aprender de otras miradas profesionales; se habilitó el uso de Facebook e Instagram, para motivar y promover el trabajo de los grupos durante el proceso de la enseñanza-aprendizaje con el AOP.

2. Cierre de ciclo. En las reuniones presenciales, el equipo de docentes organizó y planificó el evento denominado: Resultados académicos por competencias, como parte de la planeación didáctica. Esta actividad tenía como objetivo la participación de la comunidad académica con sujetos microempresarios de distintos ramos y directivos de la IE. En el evento participó el estudiantado de las cuatro licenciaturas, seleccionado a través de una audición, la cual fue coordinada por el equipo docente en actividades de logística, relaciones públicas, gestión administrativa, conducción, grabación y edición.

En este caso, la intervención propuesta, además de buscar guiar hacia la mejora de las competencias docentes, permitió al estudiantado involucrarse activamente en su aprendizaje. Lo anterior se aprecia en la planeación didáctica que incorpora un contexto complejo en términos de la resolución de algún problema, ya sea educativo, laboral o social.

Un aspecto interesante en la presentación de los resultados académicos fue la comunicación lograda a través de los PEV (portafolio de evidencias virtual), los cuales permiten un proceso de aprendizaje dentro de otro. Lo anterior se advierte en: a) portafolio de actuación docente y el b) PEV del estudiantado (Ver Figura 6). 
http://doi.org/10.15359/ree.26-1.6

http://www.una.ac.cr/educare

educare@una.ac.cr

Figura 6: Plataformas de actuación académica

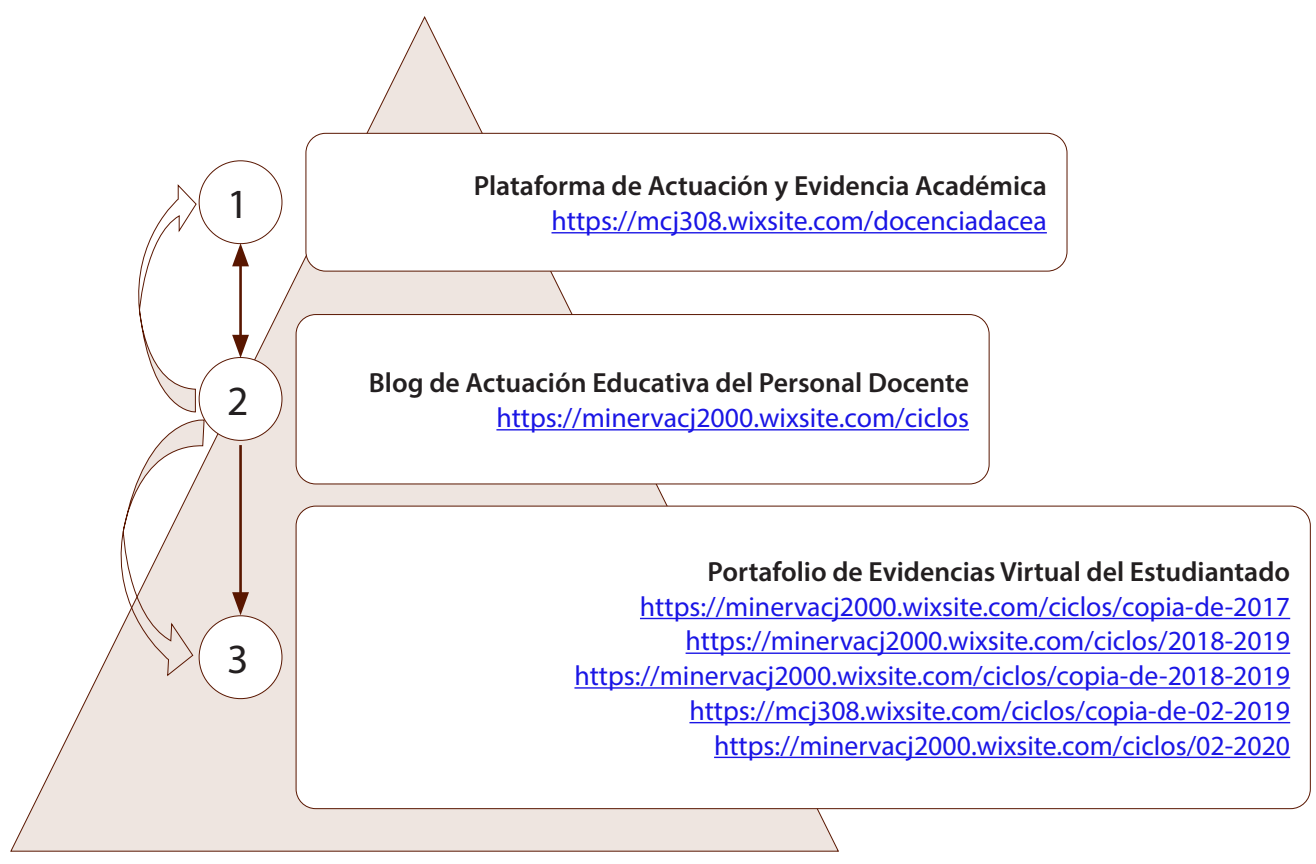

Nota: El orden de secuencia de las flechas indica la interconexión entre las plataformas. La 1 puede conectar con la 2 y viceversa. La 2 se conecta tanto con la 1 como con la 3 , pero la 3 no se conecta a la 1 y a la 2, a menos que el estudiantado decida establecer esta conexión. Un dato por destacar fue generar interés en el uso de Creative Commons.

Elaboración propia.

\section{Evidencias de la enseñanza-aprendizaje por competencias}

Derivado del punto anterior, los resultados de estos eventos se aprecian los PEV como parte del desarrollo de las competencias digitales, tanto del personal docente involucrado como del estudiantado. Los PEV muestran una identidad propia en cuanto a imagen y estructura, así como en la presentación del contenido y las evidencias desarrolladas por el estudiantado. Esto fue elaborado por el personal docente participante en los talleres formativos. Esta es una herramienta útil para integrar el aprendizaje significativo, pues conduce a la autonomía académica y genera mayor consciencia a través de la evaluación continua (Salazar-Mercado y Arévalo-Duarte, 2019).

\section{Interacción didáctica-pedagógica}

La Figura 7 comprende los atributos psicosociales relativos a la interacción que ocurre en el proceso profesionalizante. 
http://doi.org/10.15359/ree.26-1.6 http://www.una.ac.cr/educare educare@una.ac.cr

Figura 7: Interacción didáctica-pedagógica

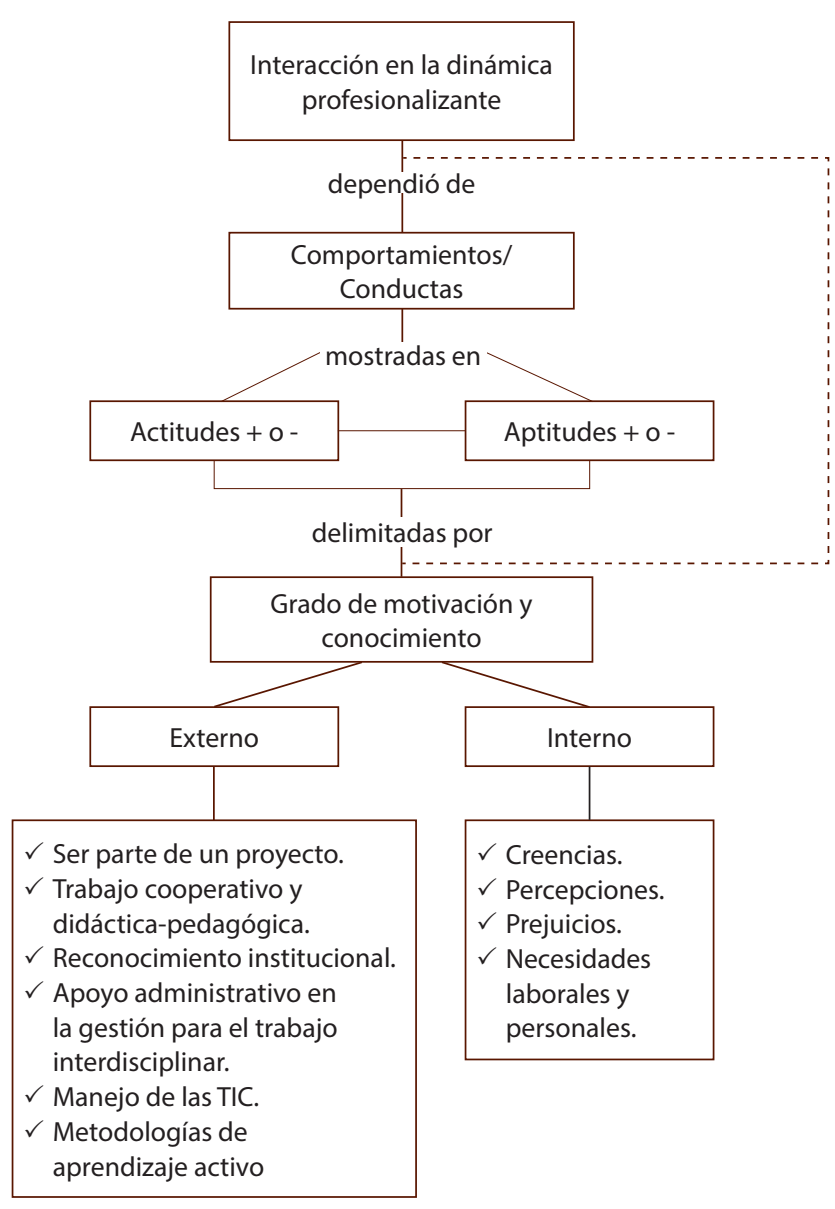

Nota: Los datos corresponden a observaciones durante el proceso profesionalizante.

Elaboración propia.

\section{La teoría en la práctica}

La revisión y análisis de la teoría se llevó a cabo con el personal docente involucrado; con la finalidad de dimensionar la complejidad y pertinencia del proceso profesionalizante.

Se utilizó el software ATLAS.ti v.9, para obtener un marco conceptual adecuado para mostrar los procesos o conjunto de operaciones relativos a la calidad educativa. Además, este tipo de actividades fomenta el desarrollo del pensamiento crítico.

En la Figura 8 se sintetiza la imbricación entre las variables teóricas emergentes que fueron recurrentes; se identificó como base la competencia gestora al direccionar y organizar el engranaje académico; esta es necesaria y significativa durante todo el proceso. 
http://doi.org/10.15359/ree.26-1.6

http://www.una.ac.cr/educare

educare@una.ac.cr

Figura 8: Imbricación de las variables identificadas en el discurso teórico

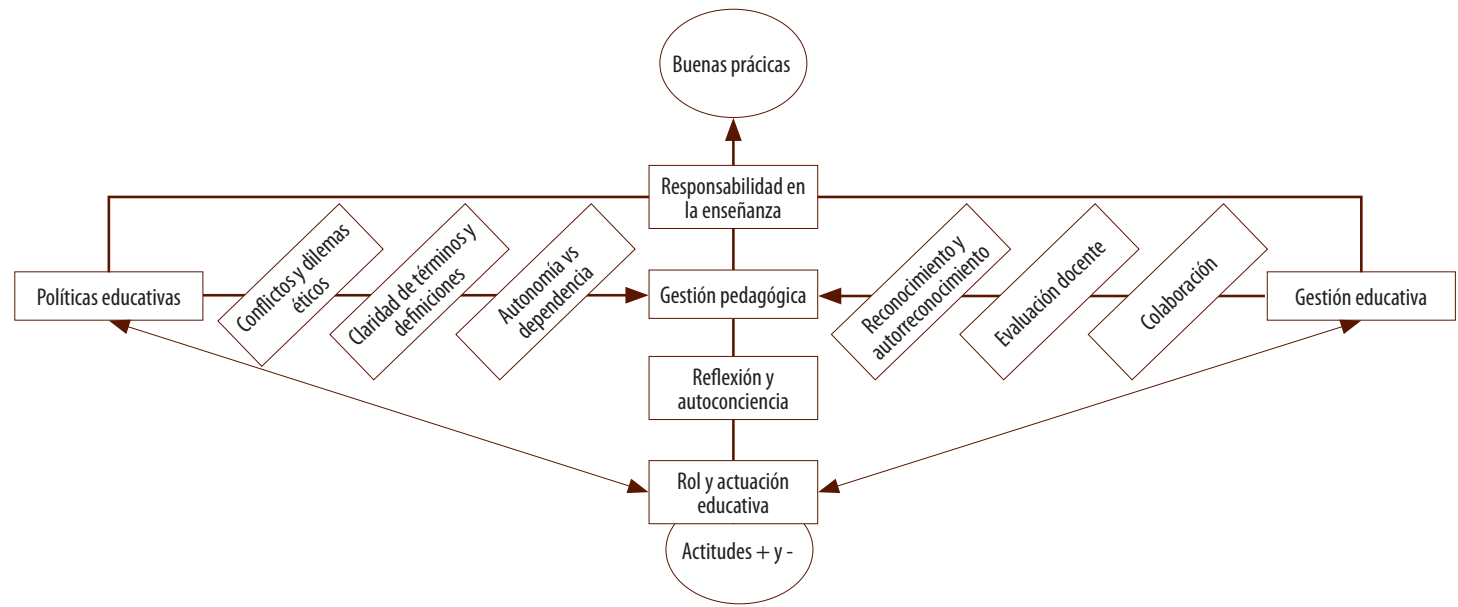

Nota: Las líneas gruesas, con una punta de flecha, contienen los elementos conceptuales de políticas y gestión educativas que incurren con la gestión pedagógica, indicando una implicación horizontal con un mismo fin, ya que son conocimientos que direccionan, organizan y resuelven en el desarrollo de un proceso educativo de calidad. La línea delgada con puntas de flechas en ambos extremos, indican la fragilidad de las actuaciones entre la docencia y los directivos, en ambos sentidos, cuando existe conflicto de intereses o pedagógicos que llevan a la disonancia comunicativa.

Elaboración propia.

El análisis teórico también ayudó a la argumentación con personal directivo-docente en turno. De los tres sujetos directivos que estuvieron durante el tiempo de aplicación del proceso profesionalizante se obtuvieron las respuestas de la Tabla 3.

Tabla 3: Acciones gestoras

\section{Acción gestora}

Permisos para contactar e invitar a personas participantes externas.

Solicitud con el personal directivo para involucrar colaboraciones con otras instancias.

Solicitud de espacios y equipamiento para: talleres, asesorías, retroalimentación didáctica-pedagógica y el trabajo reflexivo.

Solicitud de diversas constancias entregadas.

Apoyo para la comunicación de los eventos al final de cada ciclo, incluido el apoyo de radio y TV de la UJAT.

Gestión para utilería, mamparas, coffe break, conexiones, entre otros.

Total

Número de veces solicitado

Número de apoyos

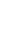

4

3

9

8

Nota: Elaboración propia. Datos obtenidos del inventario de archivos registrados por solicitud de permisos. 
http://doi.org/10.15359/ree.26-1.6

http://www.una.ac.cr/educare educare@una.ac.cr

\section{Conclusiones}

1. El proceso profesionalizante se alinea con las estrategias del modelo educativo y el plan de desarrollo divisional al promover la actualización y desarrollo de competencias pedagógicas, tecnológicas y disciplinares.

2. La organización entre docentes propicia una gestión innovadora que se manifiesta durante el trabajo colaborativo.

3. La exposición argumentada de los proyectos académicos promueve el involucramiento, apoyo y reconocimiento de las áreas administrativas. Tal exposición refiere a saberes y creencias de los maestros y las maestras.

4. El desarrollo didáctico-pedagógico del personal docente participante propició un saber y hacer interdisciplinar, el cual implica una serie de significados por parte de los maestros y las maestras.

5. La implementación de las TIC mostró la necesidad de reforzar aquellos aspectos que muestran debilidad en el diseño de su planeación didáctica. Esto revela la presencia de actitudes, como resistencia al cambio.

6. Las técnicas de aprendizaje, como el AOP, desarrollan la creatividad en la práctica educativa. El desarrollo de las competencias transversales se asocia con un trabajo colaborativo y participativo eficiente.

7. El proceso profesionalizante logra el autorreconocimiento del personal docente participante, mejora el sentir sobre todo el trabajo desarrollado. De esta manera es como se asumen los maestros y las maestras que participan en un proceso de este tipo.

8. El proceso profesionalizante favorece la adaptación a entornos educativos online. Al desarrollar las competencias docentes, los maestros y las maestras se adaptan más fácilmente a los cambios del contexto social, se asume que la educación debe resolver los problemas sociales aún insolubles.

\section{Discusión}

De acuerdo con los planteamientos de Daniels (2003) sobre la frustración que se genera al buscar la calidad educativa por la incomprensión de las instituciones con respecto a nuevos aprendizajes, es claro que el proceso profesionalizante implica gran complejidad para los maestros y las maestras y los entes directivos, en tanto no se llegue a comprender en su totalidad.

En el rol directivo-docente se fomentan los escenarios que involucran el "prácticum" en el desarrollo de las competencias (Tejada Fernández, 2020), con el objetivo principal de la agilización de gestiones académicas tanto con los diferentes actores educativos como con 
http://doi.org/10.15359/ree.26-1.6

http://www.una.ac.cr/educare

educare@una.ac.cr

aquellos externos a este. Sin embargo, en la cotidianidad se identifican comportamientos que se alejan de esta cualidad y demeritan el desarrollo de procesos de calidad.

El trabajo colaborativo y participativo entre docentes es un saber-puente para la profesionalización de las competencias docentes (Camacho Javier et al., 2018), ya que implica que ninguna propuesta educativa, por sí sola, es capaz de transformar o ser del todo exitosa si continúa prevaleciendo el protagonismo individual.

De esta manera, el acompañamiento y el tipo de interacción mostrado durante el proceso formativo (consciencia en el otro ser), es fundamental en la co-creación del conocimiento (Ponce Morales, 2017; Vygotsky,1978; Wertsch,1988). Sin embargo, lo dicho, aunque es claro y reconocido teóricamente, en la práctica la acción hacia una enseñanza-aprendizaje por competencias sigue siendo complicada y lenta por diversidad de factores imputados más a necesidades individuales de los sujetos involucrados que a necesidades educativas.

Existe coincidencia con el trabajo de Santibáñez et al. (2018), quienes reconocen la importancia de las interacciones entre la comunidad académica y de esta con el entorno social mediante un trabajo interdisciplinario. En este orden, el proceso profesionalizante provee un marco de referencia que establece pautas para vincular el ámbito académico con el ámbito empresarial.

En los productos realizados por el personal docente participante y el estudiantado se observa el desarrollo de algunas competencias señaladas por Perrenoud (2004) y Tobón (2017). Sin embargo, existe desconfianza, confusión, incertidumbre, ansiedad y poca continuidad en la metodología por proyectos, si para ello no se cuenta con el apoyo directivo.

Una gestión educativa encaminada hacia un currículo más cohesionado con la interdisciplinariedad y transdisciplinariedad implica una visión de cambio en los procesos educativos. Por lo tanto, el liderazgo proactivo, al involucrar la investigación acción colaborativa y participativa, guía hacia un saber-puente en el desarrollo de las competencias docentes; sin embargo, se requiere continuidad de este saber para innovar en la educación superior.

\section{Declaración de Material complementario}

Este artículo tiene disponible, como material complementario:

-La versión preprint del artículo en https://doi.org/10.5281/zenodo.4906991 
http://doi.org/10.15359/ree.26-1.6

http://www.una.ac.cr/educare educare@una.ac.cr

\section{Referencias}

Aberbuj, C. M., Guevara, J., y Fernández Fastuca, L. (2019). El desafío de la enseñanza en el nivel superior: Una experiencia de desarrollo profesional docente situado. RAES. Revista Argentina de Educación Superior, 11(18), 12-23. http://www.revistaraes.net/numeros. php?revista=revista18.inc

Aguilar Morales, N., Sandoval Caraveo, M. del C. y Guzmán Fernández, C. (2013). Capítulo IV. Satisfacción laboral. En D. E. Magaña Medina (Coord.), Factores organizacionales y psicosociales que inhiben el desempeño académico (pp. 83-112). UJAT. http://pcientificas. ujat.mx/index.php/pcientificas/catalog/view/98/88/348-1

Arechavala Vargas, R., y Sánchez Cervantes, C. F. (2017). Las universidades públicas mexicanas: Los retos de las transformaciones institucionales hacia la investigación y la transferencia de conocimiento. Revista de la Educación Superior, 46(184), 21-37. https://doi.org/10.1016/j. resu.2017.09.001

Asociación Nacional de Universidades e Instituciones de Educación Superior. (2018). Visión y acción 2030. Propuesta de la ANUIES para renovar la educación superior en México. Diseño y concertación de políticas públicas para impulsar el cambio institucional. Autor. https:// visionyaccion2030.anuies.mx/Vision accion2030.pdf

Bisquerra Alzina, R. (Coord.). (2009). Metodología de la investigación educativa. Editorial La Muralla.

Camacho Javier, M., López del Castillo, J. C., Reyes Cornelio, R. y Camacho Javier, D. (2018). Las competencias docentes como un saber puente en la enseñanza profesional. Visum Mundi, 2(3), 16-22. https://drive.google.com/drive/folders/1Q2Ej3A9nzQXsux7aGl61ieN7hjvh8x6V

Cañón Rodriguez, J. C. (2003). Estándares de calidad: Sus efectos en las aulas. Un ejercicio de microzonificación normativa. Universidad Nacional de Colombia.

Casanova Romero, I., Canquiz Rincón, L., Paredes Chacín, Í. e Inciarte González, A. (2018). Visión general del enfoque por competencias en Latinoamérica. Revista de Ciencias Sociales, 24(4), 114-125. https://doi.org/10.31876/rcs.v24i4.24913

Castillo-Cedeño, I., Flores-Davis, L. E., Miranda-Cervantes, G. y Murillo-León, S. (2016). Docencia universitaria saludable: Una metamorfosis impostergable. Revista Electrónica Educare, 20(2), 1-27. https://doi.org/10.15359/ree.20-2.18

Colmenares, A. M. (2012). Investigación-acción participativa: Una metodología integradora del conocimiento y la acción. Voces y Silencios: Revista Latinoamericana de Educación, 3(1), 102-115. https://doi.org/10.18175/vys3.1.2012.07 
http://doi.org/10.15359/ree.26-1.6

http://www.una.ac.cr/educare

educare@una.ac.cr

Creswell, J. W. (2008). Mixed Methods Designs. En J. W. Creswell (Autor), Educational research. Planning, conducting and evaluating cuantitative and qualitative research (3. ${ }^{a}$ ed., pp. 534-575). Merrill Prentice Hall. https://lcwu.edu.pk/ocd/cfiles/TESOL/MSTSL-505/EducationalResearchPlanningConductingandEvaluatingQuantitativeand QualitativeResearch.pdf

Daniels, H. (2003). Vygotsky y la pedagogía. Paidós. https://doi.org/10.4324/9780203469576

Díaz Barriga, F. y Rigo, M. A. (2009). Formación docente y educación basada en competencias. En Á. Valle Flores (Coord.), Formación en competencias y certificación profesional (2.a reimp., pp. 76-104). Universidad Nacional Autónoma de México. http://www.iisue.unam.mx/ publicaciones/libros/formacion-en-competencias-y-certificacion-profesional

Durán Rodríguez, R. y Estay-Niculcar, C. A. (2016). Formación en buenas prácticas docentes para la educación virtual. RIED. Revista Iberoamericana de Educación a Distancia, 19(1), 209-232. https://doi.org/10.5944/ried.19.1.13845

Freire, P. (2006). Pedagogía de la autonomía. Saberes necesarios para la práctica educativa (11. a ed.). Siglo XXI editores.

Garduño, V. (2019). Momentos clave de la profesionalización docente en México. Revista en Red, 1-10. https://www.inee.edu.mx/momentos-clave-de-la-profesionalizacion-docente-enmexico/

Hernández Sampieri, R., Fernández Collado, C. y Baptista Lucio, P. (2014). Metodología de la investigación (6. ${ }^{\mathrm{a}}$ ed.). Mac Graw Hill.

Lamoyi Bocanegra, C. L. y Pintos Blancas, M. T. (2018). La educación superior en Tabasco como factor de desarrollo económico y social. En G. Hoyos Castillo, S. E. Serrano Oswald y M. del P. Mora Cantellano (Coords.), Ciudad, género, cultura y educación en las regiones (pp. 909929). Universidad Nacional Autónoma de México; Asociación Mexicana de Ciencias para el Desarrollo Regional. http://ru.iiec.unam.mx/3957/

Magaña Medina, D. E. y Aquino Zúñiga, S. P. (2014). Evaluación docente. Experiencias en la construcción de un modelo. UJAT. https://pcientificas.ujat.mx/index.php/pcientificas/ catalog/view/34/29/123-1

Magaña Medina, D. E., y Sánchez Escobedo, P. A. (2013). Capítulo I. Síndrome de desgaste emocional. En D. E. Magaña Medina (Coord.), Factores organizacionales y psicosociales que inhiben el desempeño académico. 15-44. UJAT. http://pcientificas.ujat.mx/index.php/ pcientificas/catalog/view/98/88/348-1

Morin, E. (1990). Introducción al pensamiento complejo. Gedisa. 
http://doi.org/10.15359/ree.26-1.6 http://www.una.ac.cr/educare educare@una.ac.cr

Perrenoud, P. (2004). Diez competencias docentes para enseñar. Invitación al viaje. Grao.

Ponce Morales, M. A. (2017). Grupos interactivos en educación universitaria: Estrategia orientada al éxito para el aprendizaje con sentido. Opción, 33(84), 404-439. https:// produccioncientificaluz.org/index.php/opcion/article/view/23395

Ramírez-Díaz, J. L. (2020). El enfoque por competencias y su relevancia en la actualidad: Consideraciones desde la orientación ocupacional en contextos educativos. Revista Electrónica Educare, 24(2), 1-15. https://doi.org/10.15359/ree.24-2.23

Salazar-Mercado, S. A. y Arévalo-Duarte, M. A. (2019). Implementación del portafolio como herramienta didáctica en educación superior: Revisión de literatura. Revista Complutense de Educación, 30(4), 965-981. https://doi.org/10.5209/rced.59868

Santibáñez, L., Rubio, D. y Vázquez, M. (2018). Formación continua de docentes: Política actual en México y buenas prácticas nacionales e internacionales. INEE; BID.

Tejada Fernández, J. (2020). El prácticum en educación superior. Algunos hitos, problemáticas y retos de las tres últimas décadas. REDU. Revista de Docencia universitaria, 18(1), 105-121. https://doi.org/10.4995/redu.2020.13036

Tobón, S., Sr. (2017). Pensamiento complejo: Evaluación y desarrollo mediante proyectos formativos. Kresearch Corporation.

Vaillant,D. (2016). Trabajo colaborativo y nuevos escenarios para el desarrollo profesional docente. Docencia, 20(60), 5-13. http://www.denisevaillant.com/wp-content/uploads/2018/09/ Aprendizaje-Colaborativo-2016.pdf

Villarroel, V. A. y Bruna, D. V. (2017). Competencias pedagógicas que caracterizan a un docente universitario de excelencia: Un estudio de caso que incorpora la perspectiva de docentes y estudiantes. Formación Universitaria, 10(4), 75-96. https://dx.doi.org/10.4067/S071850062017000400008

Vygotsky, L. (1978). Pensamiento y lenguaje. Editorial Revolucionaria.

Wertsch, J. V. (1988). Vygotsky y la formación social de la mente. Paidós. 